\title{
Endoftalmitis endógenas: revisión de una serie de casos y revaloración de una entidad ocular grave
}

\author{
María A. Asencio-Egea, María Huertas-Vaquero, Rafael Carranza-González, Javier Celis-Sánchez, \\ Fernando González-del Valle, José M. Tenías-Burillo y José R. Barberá-Farré
}

\begin{abstract}
Ciudad Real, España
Alcázar de San Juan

Hospital General La Mancha

Centro

Laboratorio de Microbiología (MAA,

$\mathrm{MH}, \mathrm{RC})$.

Servicio de Oftalmología (JC, FG).

Unidad de Apoyo a la Investigación

(JMT).

Servicio de Medicina Interna (JRB)

Financiamiento: Fundación Sociosanitaria de Castilla La Mancha (PI 2009/47)
\end{abstract}

Recibido: 10 de abril de 2013 Aceptado: 16 de agosto de 2013

Correspondencia a: María Ángeles Asencio-Egea Marian_asencio@yahoo.es

\author{
Endogenous endophthalmitis: case report and brief review of a \\ serious ocular disease
}

Introduction: Endogenous endophthalmitis (EE) is a prevalent but serious disease. Our aim was to describe cases of EE, with emphasis in the risk factors and the improvement of the prognosis. Methods: A review of EE cases was done between 1996-2011 in a secondary care hospital in Spain. The reported variables were: comorbidities, isolated microorganisms, susceptibility to antimicrobial treatment and visual prognosis. Results: 9 cases of EE were analyzed. All had some underlying disease, diabetes mellitus being the most frequent. Seven of the nine cases had a history of eye injury. Extraocular source of infection was identified in 7 cases, with predominantly gastrointestinal disease. Most microorganisms were isolated from blood cultures. The visual prognosis was unfavorable in five patients and was associated with virulent microorganisms and delayed treatment. Conclusions: $\mathrm{EE}$ is a rare disease that involve immunocompromised patients with ophthalmic disease. To improve prognosis, appropriate diagnosis and early treatment is require. Therefore, we recommend funduscopy examination in patients with sepsis, risk factors and prior history of ocular disease.

Key words: Endogenous endophthalmitis; underlying diseases; prognosis; fundus examination.

Palabras clave: Endoftalmitis endógena; enfermedades subyacentes; pronóstico; fondo de ojo.

\section{Introducción}

L a endoftalmitis endógena (EE) o metastásica resulta de la diseminación hematógena de un microorganismo que atraviesa la barrera hemato-ocular desde un foco primario extraocular, siendo los orígenes más frecuentes los abscesos gastrointestinales o hepáticos, infecciones del tracto urinario, endocarditis, meningitis, neumonía e infección de piel y tejidos blandos. Su incidencia es baja, $2-10 \%$ de todos los casos de endoftalmitis ${ }^{1,2}$. Entre los factores de riesgo de la infección se incluyen las patologías crónicas, inmunodepresión, usuarios de drogas por vía parenteral (UDVP), procedimientos y técnicas invasoras y/o tratamiento antimicrobiano de amplio espectro ${ }^{3-5}$.

$\mathrm{El}$ foco causante de la bacteriemia o fungemia puede ser inaparente en las fases iniciales de la infección ${ }^{5}$, por lo que es frecuente el retraso en el diagnóstico y, por tanto, en el tratamiento; otro motivo de retraso en el tratamiento puede ser un error en el diagnóstico, siendo las causas más frecuentes de confusión: conjuntivitis, uveítis no infecciosa, iritis, celulitis, glaucoma de ángulo cerrado o endoftalmitis fúngica, sobre todo en UDVP ${ }^{1,3-5}$.

El pronóstico visual es sombrío y se relaciona fundamentalmente con el retraso en el diagnóstico, la agudeza visual (AV) a la presentación y la virulencia del patógeno, especialmente en infecciones por bacterias gramnegativas y hongos ${ }^{1,4-8}$.

El objetivo de nuestro estudio es describir los casos de EE diagnosticados en un hospital secundario, con el fin de determinar los factores de riesgo asociados, mejorar el manejo diagnóstico-terapéutico y así, el pronóstico de los pacientes.

\section{Materiales y Métodos}

Se ha realizado un estudio retrospectivo de todos los casos de EE diagnosticados en el Servicio de Oftalmología del Hospital General La Mancha Centro (Alcázar de San Juan, España) que ofrece cobertura a un área de población de 190.000 habitantes (Fuente: Instituto Nacional de Estadística, 2011) y que atiende un promedio de 14.108 consultas/año, en un período de 16 años (1996-2011). La revisión de las historias clínicas incluyó la recogida de datos demográficos, co-morbilidades asociadas, tipo de muestra/s enviada/s al laboratorio de Microbiología, microorganismo/s aislado/s, susceptibilidad a los antimicrobianos, tratamiento administrado y evolución de la infección (pronóstico visual). 
Se incluyeron en el estudio los pacientes con diagnóstico clínico de endoftalmitis endógena, definido como la presencia de células en la cámara anterior (Tyndall positivo $)^{9}$. Dependiendo de la gravedad de la infección pueden aparecer, además, los siguientes signos: fibrina en la cámara anterior y/o hipopion y/o vitritis aguda o focos vítreos o retino-coroideos ${ }^{9,10}$. Se excluyeron aquellos pacientes con historia previa de cirugía intraocular, traumatismo ocular, antecedente de ampolla de filtración o úlcera corneal el año anterior al diagnóstico de la infección. Las muestras enviadas al laboratorio de Microbiología fueron humor vítreo (HV), aguja de aspiración vítrea y hemocultivos. El HV se inoculó directamente en viales de hemocultivos pediátricos (dado el escaso volumen de la muestra) y la aguja de aspiración se introdujo en caldo de enriquecimiento tioglicolato (DIFCO, Soria Melguizo, España, S.A.). Los hemocultivos se mantuvieron cinco días a $35^{\circ} \mathrm{C}$ en el sistema automático BacT/ALERT ${ }^{\circledR}$ (BioMérieux, España, S.A.) y el tioglicolato se mantuvo siete días a $37^{\circ} \mathrm{C}$. Cuando se detectó crecimiento microbiano en los hemocultivos o en el tioglicolato, se realizó tinción de Gram y traspasos a los medios de cultivo sólidos adecuados para aislar aerobios, anaerobios y hongos. La identificación de los aislados se hizo mediante el sistema automatizado VITEK-2® (BioMérieux, España S.A.).
El estudio de la susceptibilidad a los antimicrobianos se realizó por el sistema VITEK-2® (BioMérieux, España S.A.), tiras de E-test (MIC Test Strip, Liofilchem, Italy) y el sistema comercial Sensititre ${ }^{\circledR}$ YeastOne (Trek, Diagnostic Systems, Ohio, USA) para las especies de Candida.

\section{Resultados}

El estudio incluye nueve casos diagnosticados de EE, con una mediana de edad de 70 años (rango de 1 a 85 años) y un predominio del sexo femenino ( 7 casos). Dos de ellos presentaron afectación bilateral. Las manifestaciones clínicas fueron las siguientes: dolor ocular, conjuntiva congestiva, párpados hinchados y brusca disminución de la agudeza visual.

Las muestras enviadas al laboratorio de Microbiología, los distintos aislados microbianos, su sensibilidad a los antimicrobianos, el tratamiento administrado, la evolución de los pacientes y la AV final se muestran en la Tabla 1. El hemocultivo fue positivo en cuatro casos, recuperándose dos bacilos gramnegativos, una bacteria grampositiva y una levadura de la especie Candida, respectivamente. Únicamente se aisló un microorganismo, Candida albicans, en las muestras intraoculares, cuyo rendimiento

\begin{tabular}{|c|c|c|c|c|c|c|}
\hline Caso & Muestras & Microorganismo/ R & $\begin{array}{l}\text { Vitrectomía } \\
\text { pars plana }\end{array}$ & Tratamiento y vías & Complicaciones & $\begin{array}{l}\text { Agudeza } \\
\text { visual final }\end{array}$ \\
\hline 1 & $\begin{array}{l}\text { Hemocultivo }(-), \mathrm{HV}(-) \text {, } \\
\text { urocultivo }(-)\end{array}$ & Sin aislamiento & No & $\begin{array}{l}\text { Vancomicina }+ \text { ceftazidima }(\operatorname{Itv} \times 2, T) \\
\text { gentamicina }(T)\end{array}$ & - & $<0,05$ \\
\hline 2 & Hemocultivo $(+), \mathrm{HV}(-)$ & $\begin{array}{l}\text { Enterobacter cloacae } \\
\text { R: ampicilina, amoxicilina/ } \\
\text { ác. clavulánico, cefazolina }\end{array}$ & No & Vancomicina + ceftazidima (Itv) & - & $\begin{array}{l}<0,1 \\
<0,1\end{array}$ \\
\hline 3 & Hemocultivo $(+)$ & Candida sp. & Sí & Fluconazol $(\mathrm{IV}, \mathrm{O})^{*}$, gentamicina $(\mathrm{T})$ & $\begin{array}{l}\text { Atalamia, desprendimiento } \\
\text { de retina, amaurosis }\end{array}$ & NPL \\
\hline 4 & Hemocultivo (-), LCR (-) & Sin aislamiento & No & Cefotaxima (IV), rifampicina (T) & & 0,7 \\
\hline 5 & Ninguna & Sin aislamiento & No & $\begin{array}{l}\text { Vancomicina + ceftazidima }(I V, T) \text {, } \\
\text { quinolona }(O) \text {, tetraciclina }(T)\end{array}$ & Enucleación & - \\
\hline 6 & $\begin{array}{l}\text { Hemocultivo }(+) \text {, } \\
\text { urocultivo }(+)\end{array}$ & $\begin{array}{l}\text { Escherichia coli } \\
\text { R: ampicilina, quinolona }\end{array}$ & No & Ceftriaxona (IV), tobramicina (T) & - & 0,4 \\
\hline 7 & Ninguna & Sin aislamiento & No & $\begin{array}{l}\text { Vancomicina + ceftazidima (Itv, T), } \\
\text { quinolona }(T)\end{array}$ & Atalamia & - \\
\hline 8 & $\begin{array}{l}\text { Hemocultivo }(+), \\
\text { aguja }(-), \\
\text { exudado de herida** }(1)\end{array}$ & $\begin{array}{l}\text { Staphylococcus aureus } \\
\text { R: penicilina }\end{array}$ & Sí & $\begin{array}{l}\text { Vancomicina (Itv, T), ceftazidima (T), } \\
\text { cloxacilina (IV), quinolona (IV, O) }\end{array}$ & - & 0,7 \\
\hline 9 & Hemocultivo $(-), \mathrm{HV}(+)$ & $\begin{array}{l}\text { Candida albicans } \\
\text { (Ojo derecho) }\end{array}$ & Sí & Anfotericina B (Itv), voriconazol (Itv x 2,0) & - & $\begin{array}{l}0,8 \\
0,7\end{array}$ \\
\hline \multicolumn{7}{|c|}{$\begin{array}{l}\text { R: resistencia antibiótica. VPP: vitrectomía pars plana. Itv x 2: repetición de inyecciones intravítreas. HV: humor vítreo. NPL: no percepción de luz. Tratamiento y vías: Ivt: inyección } \\
\text { intravítrea. T: administración tópica (colirios). IV: administración intravenosa. O: administración oral. *No siguió el tratamiento. **en el exudado de herida (pie diabético); se } \\
\text { obtuvo crecimiento de S. aureus y Pseudomonas aeruginosa, mientras que en el hemocultivo creció solamente S. aureus; hubo que amputar dos dedos del pie, considerando } \\
\text { esta necrosis como el foco de infección más probable. En los casos } 5 \text { y } 7 \text { el ojo estaba amaurótico previamente a la infección. }\end{array}$} \\
\hline
\end{tabular}




\begin{tabular}{|c|c|c|c|c|c|c|c|c|}
\hline Caso & $\begin{array}{l}\text { Edad } \\
\text { (años)/ } \\
\text { Sexo }\end{array}$ & Ojo & Factor de riesgo & $\begin{array}{l}\text { Antecedente } \\
\text { oftalmológico }\end{array}$ & Enfermedades subyacentes & Foco & $\begin{array}{l}\text { Diagnóstico } \\
\text { erróneo }\end{array}$ & $\begin{array}{l}\text { Retraso en } \\
\text { tratamiento }\end{array}$ \\
\hline 1 & $70 / F$ & D & ICCC & No & ICCC, HTA & Biliar & No & No \\
\hline 2 & $75 / F$ & $D, I$ & DMNID, neoplasia & Retinopatía diabética & $\begin{array}{l}\text { DMNID, HTA, EPOC, cáncer de } \\
\text { páncreas }\end{array}$ & Gastrointestinal & No & 5 días \\
\hline 3 & $23 / M$ & D & $\begin{array}{l}\text { Alcoholismo } \\
\text { UDVP }\end{array}$ & Uveítis de repetición & $\begin{array}{l}\text { VHC, alcoholismo, foliculitis } \\
\text { cuero cabelludo y espalda }\end{array}$ & Drogadicción IV & No & $\mathrm{Si}^{*}$ \\
\hline 4 & $1 / \mathrm{F}$ & 1 & Prematuridad & $\begin{array}{l}\text { Retinopatía del } \\
\text { prematuro }\end{array}$ & $\begin{array}{l}\text { Prematura ( } 34 \text { semanas), } \\
\text { colestasis, estenosis aórtica y } \\
\text { meningitis con septicemia }\end{array}$ & SNC & No & No \\
\hline 5 & $85 / F$ & D & DMNID & $\begin{array}{l}\text { Retinopatía diabética } \\
\text { glaucoma** }\end{array}$ & DMNID, HTA & No conocido & No & 4 días \\
\hline 6 & $75 / M$ & D & $\begin{array}{l}\text { Neutropenia } \\
\text { Catéter ureteral }\end{array}$ & Queratitis post-herpética & $\begin{array}{l}\text { Sepsis de origen urinario (1), } \\
\text { neutro y trombopenia }\end{array}$ & Tracto urinario & No & No \\
\hline 7 & $64 / F$ & । & DMNID & $\begin{array}{l}\text { Retinopatía diabética, } \\
\text { glaucoma** }\end{array}$ & DMNID, HTA, dislipemia & No conocido & No & No \\
\hline 8 & $48 / \mathrm{F}$ & । & DMNID & Retinopatía diabética & DMID, IRC, ICCC & IPTB & $\begin{array}{l}\text { Endoftalmitis } \\
\text { candidiásica }\end{array}$ & No \\
\hline 9 & $82 / \mathrm{F}$ & $D, I$ & $\begin{array}{l}\text { Cardiopatía } \\
\text { Nutrición parenteral }\end{array}$ & No & $\begin{array}{l}\text { Cardiopatía, HTA, } \\
\text { colecistopancreatitis aguda }\end{array}$ & $\begin{array}{l}\text { Gastrointestinal } \\
\text { Catéter nutrición parenteral }\end{array}$ & No & 7 días \\
\hline $\begin{array}{l}\text { M: ma } \\
\text { depen } \\
\text { diabet } \\
\text { el fact }\end{array}$ & $\begin{array}{l}\text { ulino. F: } f \\
\text { ente. EPO } \\
\text { mellitus i } \\
\text { de riesgo }\end{array}$ & emenir & endiente. IRC: insufi & $\begin{array}{l}\text { izquierdo. ICCC: insuficienc } \\
\text { uctiva crónica. UDVP: usuari } \\
\text { encia renal crónica. IPTB: inf } \\
\text { Jropatía obstructiva secund }\end{array}$ & $\begin{array}{l}\text { a cardiaca congestiva crónica. } \\
\text { de drogas por vía parenteral. V } \\
\text { cción de piel y tejidos blandos. }\end{array}$ & $\begin{array}{l}\text { hipertensión arterial. DMNID } \\
\text { virus de la hepatitis C. SNC: } \\
\text { realizó el tratamiento. }{ }^{* *} \text { Ojo } \\
\text { osis infectada que evolucionó }\end{array}$ & $\begin{array}{l}\text { diabetes mellitu } \\
\text { tema nervioso } \\
\text { naurótico. En es } \\
\text { cuadro séptico }\end{array}$ & $\begin{array}{l}\text { us no insulino- } \\
\text { central. DMID: } \\
\text { stos dos casos, }\end{array}$ \\
\hline
\end{tabular}

fue de $25 \%$. Todos los microorganismos aislados fueron sensibles a los antimicrobianos administrados. Tres de los cinco casos con aislamiento microbiano presentaron una AV residual menor de 0,5 .

En la Tabla 2 se muestran los factores de riesgo y la probable fuente de infección de cada uno de los casos diagnosticados. Todos los pacientes presentaron alguna enfermedad predisponente, siendo diabetes mellitus la más frecuente (4 casos), y 7 de los 9 pacientes presentaban, además, un antecedente de patología oftálmica (no se incluyen los defectos de refracción). Cuatro pacientes experimentaron un retraso en el tratamiento y tres de ellos presentaron una AV final $<0,1$. Sólo logramos definir el posible foco de infección en siete casos: aparato gastrointestinal (quiste de colédoco, cáncer pancreático y colecisto-pancreatitis con nutrición parenteral), sistema nervioso central (meningitis meningocóccica sin confirmación microbiológica), tracto urinario, úlcera de pie diabético y la solución para inyectarse la droga en paciente UDVP.

Los antimicrobianos utilizados más frecuentemente fueron vancomicina y ceftazidima como tratamiento único, o generalmente asociados con quinolonas. Sólo dos de los nueve casos recibieron tratamiento combi- nado de antimicrobianos intravenosos e intravítreos, presentando ambos pacientes una AV final $>0,1$; tres pacientes recibieron inyecciones intravítreas, todos con una AV final menor de 0,1 , y cuatro pacientes recibieron tratamiento sistémico (la mitad de ellos con AV final $<0,1$, incluyendo la única enucleación). La mayoría de los casos (7 pacientes) recibió colirios fortificados horarios. En dos casos fue necesario administrar una segunda inyección intravítrea a las $48 \mathrm{~h}$ (casos 1 y 9). Únicamente se practicó vitrectomía pars plana (VPP) en tres casos, incluyendo las dos endoftalmitis candidiásicas.

La AV global fue menor de 0,1 en cinco casos (se han incluido el caso sin percepción de luz y el ojo enucleado), y mayor de 0,5 en tres de los nueve. Se produjo una defunción en un paciente con cáncer terminal que no quiso colaborar en las exploraciones oftalmológicas ni seguir el tratamiento indicado.

\section{Discusión}

Actualmente las EE son poco comunes, aunque suponen una de las principales causas infecciosas de pérdida visual. Nuestro estudio incluye nueve casos en 16 años 
$y$, al igual que en la mayoría de estudios revisados, todos nuestros pacientes presentaron al menos una enfermedad subyacente, predominando la diabetes mellitus ${ }^{3-5,7}$. De la misma manera, el pronóstico visual fue pobre y se relacionó con el aislamiento de microorganismos virulentos ${ }^{4-8}$ y el retraso en el tratamiento ${ }^{1}$. Sólo dos casos presentaron afectación bilateral, que suele ser más frecuente en series con predominio de agentes etiológicos fúngicos ${ }^{5,7,8}$; en nuestra serie, solamente uno de los dos casos fue de etiología fúngica.

El hemocultivo fue más útil que las muestras vítreas en la pesquisa de un patógeno, coincidiendo con otros autores de series más numerosas ${ }^{4,6}$. Destacamos que el número de muestras intraoculares obtenidas en nuestra serie fue especialmente bajo ( 4 de 9 casos) y aunque su rendimiento se ve limitado, sobre todo, por el bajo volumen que se puede obtener, creemos que los médicos clínicos han de tomar conciencia de la relevancia de identificar el agente etiológico, ya bien en el hemocultivo, en muestras vítreas y/o en cualquier otra muestra relacionada con el foco de infección, lo que permite orientar un tratamiento precoz. Asimismo, es importante que exista una buena comunicación entre médicos clínicos y microbiólogos, quienes guiarán la elección de los medios de cultivo más apropiados o la posibilidad de realizar un examen microscópico cuando la cantidad de muestra lo permita. Las técnicas de biología molecular como la $\mathrm{RPC}^{11}$, pueden aminorar el problema de los falsos negativos del cultivo por presentar mayor sensibilidad y rapidez, si bien, la RPC no ha de sustituir al cultivo convencional, pues la recuperación del aislado nos permite realizar un antibiograma específico o bien, estudios epidemiológicos. Por otra parte, conocer el patrón de resistencia en un área determinada ayuda a elegir un tratamiento empírico más adecuado. Además, la RPC no está exenta de inconvenientes como la obtención de falsos positivos debido a la posible contaminación con la microbiota ocular externa del paciente al tomar la muestra o su limitación en infecciones mixtas (poco frecuentes en los casos de EE). Actualmente, en infecciones como la EE se aplica la RPC universal (RPC 16S ARNr) que, con una sola reacción de RPC detecta la mayoría de las bacterias que pueden causar la infección ${ }^{11,12}$.

La diabetes mellitus es un factor reconocido de $\mathrm{EE}^{3-5,7}$ que aparece en 4 de los 9 casos del estudio. La prematuridad, aunque menos conocido, es también un factor de riesgo, tanto por el bajo peso al nacer $(<1.500 \mathrm{~g})^{13}$ como por la probabilidad de desarrollar retinopatía del prematuro $^{14}$, a su vez factor predisponente por favorecer la isquemia responsable de la neovascularización de los vasos, de modo similar al que acontece en la retinopatía diabética ${ }^{15}$.

La EE, a diferencia de las otras formas de endoftalmitis, está causada con mayor frecuencia por hongos que por bacterias, siendo Candida spp. el agente aislado en aproximadamente $35 \%$ de todos los casos $^{10}$. Dentro de las especies bacterianas destacan Staphylococcus aureus, Streptococcus spp, Neisseria meningitidis, enterobacterias (con predominio en el este asiático) $)^{4,6}$ y Pseudomonas aeruginosa, que está emergiendo entre los pacientes más graves ${ }^{16}$. En pacientes inmunodeprimidos o UDVP se aíslan Candida spp. ${ }^{10}$, Aspergillus spp con elevada mortalidad $^{17}$ y otros hongos filamentosos ${ }^{18}$, Listeria monocytogenes ${ }^{19}$ o Bacillus cereus, principalmente en UDVP por contaminación de la solución utilizada para inyectarse la droga ${ }^{1}$.

Todos los aislados de nuestra serie fueron sensibles a los antimicrobianos administrados, por lo que el mal pronóstico visual de nuestros casos no puede relacionarse con un fracaso terapéutico. Únicamente el caso 9 presentó intolerancia a anfotericina $\mathrm{B}$, obligando a sustituirla por otro antifúngico.

Vancomicina y ceftazidima constituyen una buena elección para el tratamiento empírico de la EE bacteriana, mientras que para el tratamiento empírico de endoftalmitis candidiásica se recomienda anfotericina B liposomal, y fluconazol como segundo escalón terapéutico, cuando aparecen infiltrados corio-retinales algodonosos junto con vitritis o iritis, especialmente en UDVP y hospederos inmunodeprimidos ${ }^{20}$. La realización de VPP inmediata se recomienda en casos graves, sin respuesta al tratamiento intravítreo o cuando la AV esté reducida a percepción de $\mathrm{luz}^{21}$, pues además de mejorar el pronóstico visual, podría evitar la enucleación ${ }^{4,6}$. De hecho, en nuestra serie la VPP se practicó en los casos más agresivos, (casos 3, 8 y 9), alcanzando una AV final $>0,1$ en dos de los tres casos. Por esta razón, se justifica la realización de VPP precoz en las endoftalmitis fúngicas, cuya evolución suele ser más tórpida que las bacterianas ${ }^{5,8}$. El caso 3, paciente UDVP y alcohólico, con endoftalmitis candidiásica, no quiso seguir tratamiento ocular alguno ni someterse a cirugía hasta un mes después del diagnóstico de la infección, presentando diversas complicaciones que derivaron en amaurosis.

Por otra parte, se localizó el probable foco extraocular en la mayoría de los casos, siendo de mayor frecuencia la fuente gastrointestinal ( 3 de los 7 casos en los que se identificó); en el caso 9 es discutible si el foco de la infección fue la colecistopancreatitis o el catéter de nutrición parenteral, ya que la levadura sólo se aisló en un hemocultivo de venopunción periférica. Aunque la bibliografía muestra una gran variabilidad, con cifras que oscilan entre 50 y $93 \% \%^{3-5}$, estimamos de gran valor localizar el foco primario por permitir un tratamiento integral de la infección. A pesar de la controversia en relación a la utilidad del tratamiento sistémico por no alcanzar niveles terapéuticos en el ojo ${ }^{4}$, consideramos primordial combinar tratamiento sistémico con intravítreo a fin de tratar el foco de infección y la septicemia, consiguiendo así una mayor eficacia terapéutica ${ }^{3,6}$. De hecho, la evolución de 
nuestros pacientes fue peor cuando se utilizó una única vía de administración. Tras varias décadas de avances diagnósticos y terapéuticos, el pronóstico visual de esta afección sigue siendo tan pobre como el presentado por otros autores en los últimos 30 años ${ }^{1,3,4,6-8,10}$. La tasa de mortalidad relacionada con el foco de nuestro estudio fue de $11 \%$, el doble que la hallada en la revisión de Jackson ${ }^{4}$ y muy inferior al $32 \%$ encontrado en un estudio prospectivo de pacientes con bacteriemia ${ }^{21}$.

Debido a que en las fases iniciales de la infección el foco puede no ser aparente y que entre 16 y $50 \%$ de los casos las EE se diagnostican erróneamente ${ }^{1,3-5}$, es frecuente el retraso del tratamiento adecuado. En nuestra serie, el único caso que requirió enucleación presentó un retraso en el tratamiento de cuatro días. Por ello, independientemente de la presentación de síntomas oculares, se recomienda realizar un fondo de ojo a los pacientes con candidemia ${ }^{22,23}$. En base a nuestros datos, consideramos conveniente incluir el estudio del fondo de ojo o un examen oftalmológico diario en los protocolos clínicos de actuación en los pacientes con bacteriemia, factores predisponentes de EE y patología ocular inflamatoria o isquémica previa ( 7 casos de 9 en nuestra serie). Es conocido que durante un proceso de bacteriemia las metástasis sépticas suelen asentar en zonas anatómicas debilitadas o con material protésico, por lo que en un paciente séptico con historia de lesión ocular previa debiéramos excluir la posibilidad de implante séptico en uno o ambos ojos.

Este estudio presenta las limitaciones propias de los estudios retrospectivos, como es el mayor riesgo de presentar sesgos de información. Por otra parte, la información recogida en las historias clínicas puede no reflejar fielmente la actuación realizada. Para obtener resultados más significativos sería necesaria una serie con un mayor número de casos.

En conclusión, la EE es una enfermedad poco frecuente que afecta a pacientes con factores inmunosupresores y cuyo pronóstico visual es pobre, relacionado fundamentalmente con microorganismos virulentos y el retraso en el tratamiento adecuado. Dado que el pronóstico se asocia a un tratamiento precoz que incluye antimicrobianos intravítreos y sistémicos, el diagnóstico temprano de la infección supone un reto para médicos clínicos y microbiólogos. Por esta razón, recomendamos la realización de un fondo de ojo o examen oftalmológico diario a pacientes con sepsis y factores de riesgo de EE, sobre todo si presentan antecedentes de patología ocular inflamatoria o isquémica, son prematuros o si la gravedad del estado general del paciente impide su autoevaluación ocular.

Agradecimientos. A la Fundación Sociosanitaria de Castilla La Mancha por el apoyo económico para este estudio.

\section{Resumen}

Introducción: La endoftalmitis endógena (EE) es una patología poco prevalente aunque grave. Nuestro objetivo es describir los casos de EE diagnosticados en un hospital secundario español, con particular atención a los factores de riesgo y la posible mejora del pronóstico. Material y Métodos: Revisamos las historias clínicas de los pacientes diagnosticados de EE entre 1996-2011. Las variables recogidas fueron: co-morbilidades, microorganismo/s aislados y su susceptibilidad a los antimicrobianos, tratamiento administrado y pronóstico visual. Resultados: Se estudiaron nueve casos de EE. Todos presentaban alguna enfermedad de base, siendo diabetes mellitus la más frecuente. Siete de los nueve casos presentaron antecedentes de lesión ocular. La probable fuente extraocular se identificó en siete casos, predominando el foco gastrointestinal. La mayoría de microorganismos se aisló de hemocultivos. El pronóstico visual fue desfavorable en cinco pacientes, asociándose a microorganismos virulentos y al retraso terapéutico. Conclusiones: La EE es una enfermedad inusual que afecta a pacientes con inmunidad disminuida y antecedentes oftalmológicos. Para mejorar el pronóstico se requiere un diagnóstico acertado y un tratamiento precoz, todo un reto para médicos clínicos y microbiólogos. Por ello, recomendamos realizar un fondo de ojo a los pacientes con sepsis, factores de riesgo de EE y antecedentes de patología ocular.

\section{Referencias bibliográfícas}

1.- Greenwald M J, Wohl L G, Sell C H. Metastatic bacterial endophthalmitis: a contemporary reappraisal. Surv Ophthalmol 1986; 31 (2): 81-101.

2.- Shrader S K, Band J D, Lauter C B, Murphy P. The clinical spectrum of endophthalmitis: incidence, predisposing factors, and features influencing outcome. J Infect Dis 1990; 162 (1): 115-20.

3.- Okada A A, Johnson R P, Liles W C,
D’Amico D J, Baker A S. Endogenous bacterial endophthalmitis. Report of a ten-year retrospective study. Ophthalmology 1994; 101 (5): 832-8.

4.- Jackson T L, Eykyn S J, Graham E M, Stanford M R. Endogenous bacterial endophthalmitis: a 17-year prospective series and review of 267 reported cases. Surv Ophthalmol 2003; 48 (4): 403-23.

5.- Binder M I, Chua J, Kaiser P K, Procop G W, Isada C M. Endogenous endophthalmitis: an 18-year review of culture-positive cases at a tertiary care center. Medicine (Baltimore) 2003; 82 (2): 97-105.

6.- Wong J S, Chan T K, Lee H M, Chee S P. Endogenous bacterial endophthalmitis: an east Asian experience and a reappraisal of a severe ocular affliction. Ophthalmology 2000; 107 (8): 1483-91.

7.- $\quad$ Leibovitch I, Lai T, Raymond G, Zadeh R, Nathan F, Selva D. Endogenous endophthalmitis: a 13-year review at a tertiary hospital in South Australia. Scand J Infect Dis 2005; 37 (3): 184-9. 
8.- Schiedler V, Scott I U, Flynn H W Jr, Davis J L, Benz M S, Miller D. Culture-proven endogenous endophthalmitis: clinical features and visual acuity outcomes. Am J Ophthalmol 2004; 137 (4): 725-31.

9.- Jabs D A, Nussenblatt R B, Ronsembaum J T. Standardization of uveitis nomenclature for reporting clinical data. Results of first international workshop. J Ophthalmol 2005; 140: 509-16.

10.- Conell P P, $\mathrm{O}^{\prime}$ Neill E C, Fabinyi D, Islam F M, Buttery R, McCombe M, et al. Endogenous endophthalmitis: 10-year experience at a tertiary referral centre. Eye 2011; 25: 66-72.

11.- Gerkowicz M, Pietraś-Trzpiel M, KoziołMontewka M, Kosior-Jarecka E, Latalska M, Paluch-Oleś J. Application of polymerase chain reaction method (PCR) in diagnosis of endophthalmitis. Klin Oczna 2006; 108: 319-22.

12.- Knox C M, Cevallos V, Margolis T P, Dean D. Identification of bacterial pathogens in patients with endophthalmitis by $16 \mathrm{~S}$ ribosomal DNA typing. Am J Ophthalmol 1999; 128: 511-12.

13.- Basu S, Kumar A, Kapoor K, Bagri N K, Chandra A. Neonatal endogenous endophthalmitis: a report of six cases. Pediatrics 2013; 131: e1292-7.

14.- Moshfeghi A A, Charalel R A, HernandezBoussard T, Morton J M, Moshfeghi D M. Declining incidence of neonatal endophthalmitis in the United States. Am J Ophthalmol 2011; 151: 59-65.

15.- Palmer E, Patz A, Phelps D, Spencer R: "Retinopaty of Prematurity". In: Ryan S J, ed. $2^{\circ}$ ed 1994, vol 2, St. Louis, Mosby. "Retina" Chap 87, p. 1473-98.

16.- Reedy J S, Wood K E. Endogenous Pseudomonas aeruginosa endophthalmitis: a case report and literature review. Intensive Care Med 2000; 26 (9): 1386-9.

17.- Hunt K E, Glasgow B J. Aspergillus endophthalmitis. An unrecognized endemic disease in orthotopic liver transplantation. Ophthalmology 1996; 103 (5): 757-67.

18.- Glasgow B J, Engstrom R E Jr, Holland G N, Kreiger A E, Wool M G. Bilateral endogenous Fusarium endophthalmitis associated with acquired immunodeficiency syndrome. Arch Ophthalmol 1996; 114 (7): 873-7.

19.- Betriu C, Fuentemilla S, Méndez R, Picazo J J, García-Sánchez J. Endophthalmitis caused by
Listeria monocytogenes. J Clin Microbiol 2001; 39 (7): 2742-4.

20.- Mensa J G, Gatell J M, García-Sánchez J E, Letang E, López-Suñé E, Marco F. Endoftalmitis. In: Zamora E, editor. Guía de Terapéutica Antimicrobiana 2012. 22 ${ }^{\mathrm{a}}$ ed. Barcelona: Antares; 2012. p. 469-70.

21.- Bouza E, Cobo-Soriano R, RodríguezCreixems M, Muñoz P, Suárez-Leoz M, Cortés C. A prospective search for ocular lesions in hospitalized patients with significant bacteremia. Clin Infect Dis 2000; 30 (2): 306-12.

22.- Pappas P G, Kauffman C A, Andes D, Benjamin D K Jr., Calandra T F, Edwards J E Jr, et al. Clinical practice guidelines for the management of candidiasis: 2009 update by the Infectious Diseases Society of America. Clin Infect Dis 2009; 48: 503-35.

23.- Recomendaciones sobre el tratamiento de la candidiasis invasiva y otras infecciones por levaduras de la Sociedad Española de Enfermedades Infecciosas y Microbiología Clínica (SEIMC). Actualización 2011. Enferm Infecc Microbiol Clin 2011; 29: 345-61. 